\title{
Hydrogen and radiation induced effects on performances of Raman fiber-based temperature sensors
}

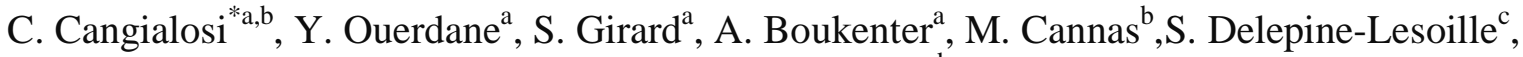 \\ J. Bertrand ${ }^{\mathrm{c}}$, P. Paillet ${ }^{\mathrm{d}}$, \\ ${ }^{a}$ Laboratoire Hubert Curien, Université de Lyon, CNRS UMR 5516, 42000 Saint-Etienne, France \\ ${ }^{\mathrm{b}}$ Dipartimento di Fisica e Chimica, Università degli studi di Palermo, I-90123 Palermo, Italia \\ ${ }^{c}$ National Radioactive Waste Management Agency (Andra), 1-7 rue Jean Monnet, Parc de la Croix \\ blanche, 92298 Chatenay-Malabry, France \\ ${ }^{\mathrm{d}}$ CEA DAM DIF, F91297 Arpajon Cedex, France
}

\begin{abstract}
Raman Distributed Temperature Sensors (RDTS) offer exceptional advantages for the monitoring of the envisioned French deep geological repository for nuclear wastes, called Cigéo. Here, we present experimental studies on how the performances of RDTS evolve in harsh environments like those associated with $\mathrm{H}_{2}$ or $\gamma$-rays. Both of them are shown to strongly affect the temperature measurements made with RDTS. We showed that by adapting the characteristics of the used fiber for the sensing, we could limit its degradation but that additional hardening by system studies will have to be developed before integration of RDTS in Cigéo.
\end{abstract}

Keywords: Distributed temperature sensor, RDTS, optical fibers, hydrogen loading, radiation, nuclear waste.

\section{INTRODUCTION}

Raman Distributed Temperature Sensors (RDTS) are intrinsic optical fiber sensors, in which the optical fiber is not only a transmission medium but also a sensing material. It acts as a transducer converting temperature variation into optical power intensity change. These systems derive the location along the multimode fiber from the time of flight of the probe pulse using optical time domain reflectometry techniques. Their temperature sensitivity is achieved through the modulation of the backscattered intensity, owing to the phenomenon of spontaneous Raman scattering. A high power probe laser launches a pulsed optical signal in the optical fiber at the probe frequency $v_{0}$. Only a small fraction of the probing power is transferred to frequencies downshifted or upshifted, namely the Stokes and anti-Stokes frequencies, and is backscattered to the fiber input. The Raman component of the backscattered light is caused by thermally driven molecular vibrations and can be used to obtain information about the temperature distribution along the fiber. The RDTS system used for this work is based on the measurement of the two Raman components from one end of the fiber (open-ended RDTS). Since the intensity of the AntiStokes line is more sensible to the temperature than the intensity of the Stokes line, in this kind of sensor the temperature is calculated according the equation:

$$
T=\frac{h \Delta v}{k \ln \left[\left(\frac{v_{a s}}{v_{s}}\right)^{4} \cdot \frac{1}{R}\right]}
$$

Despite Stokes normalization, this measurement is sensitive to variations of the fiber attenuation if this is not constant over the $100 \mathrm{~nm}$ span (between S and AS lines at 1064nm). It is the case of bending losses, and radiation-induced losses. In fact, while the laser pulse propagates in the fiber, the optical signal is attenuated by a factor $\exp (-\alpha z)$ where $\alpha$ is the fiber attenuation coefficient at the pump frequency $v_{\mathrm{p}}$. The backscattered light also undergoes an attenuation $\alpha_{\mathrm{s}}$ and $\alpha_{\mathrm{as}}$, for the Stokes and the anti-Stokes signals respectively.

*chiara.cangialosi@univ-st-etienne.fr; phone 0033-477915795.

23rd International Conference on Optical Fibre Sensors, edited by José Miguel López-Higuera,

Julian Jones, Manuel López-Amo, José Luis Santos, Proc. of SPIE Vol. 9157, 91576U

(C) 2014 SPIE · CCC code: 0277-786X/14/\$18 · doi: 10.1117/12.2059628 
If we take into account attenuation, we obtain $R(T, z)$ as a function of the position $\mathrm{z}$ of the scattering element du

$$
R(T, z)=\left(\frac{v_{a s}}{v_{s}}\right)^{4} e^{-\frac{h \Delta v}{k T(z)}} e^{-\int_{0}^{z}\left(a_{a s}(u)-a_{s}(u)\right) d u}
$$

where $\mathrm{z}$ is the distance from the fiber input. We'll see that the assumption that is commonly used in single-ended RDTS systems that $\left(\alpha_{a s}-\alpha_{s}\right)$ is constant with time and/or uniform along the length of a fiber is frequently inappropriate and especially so where the fiber is subjected to environment where its transmission is likely to be degraded. This can be the case in presence of ionizing radiation and hydrogen release like for the Cigéo applications ${ }^{1}$. If the measurements are not compensated for the differential attenuation $\left(\alpha_{a s}-\alpha_{s}\right)$, the measurement error increases with the distance ${ }^{2,3}$. The goal of this paper is to investigate $\mathrm{H}_{2}$ or gamma radiation effects on sensors performances, in view of their application in the storage cell. We'll show that both treatments, gamma-radiation and $\mathrm{H}_{2}$-loading in standard fibers, affect the measurements Raman leading to an incorrect reading of temperature. This problem is mainly explained by $\gamma$-ray or $\mathrm{H}_{2}$ induced differential attenuation (RIA and HIR, respectively) for the Raman Stokes and anti-Stokes lines.

\section{EXPERIMENTAL PROCEDURE}

\subsection{Experimental set-up and method}

All measurements were performed using a commercially open-ended Sentinel DTS-SR Raman distributed temperature sensor from Sensornet ${ }^{4}$ provided with 8 channel multiplexer. The device is linked to multimode optical fibers through standard E2000 connectors. The best instrument claimed performances are: probe frequency of $1064 \mathrm{~nm}, 0.01^{\circ} \mathrm{C}$ of accuracy (for distance $<10 \mathrm{~km}$ ), spatial resolution of $1 \mathrm{~m}$ and longest range (continuous monitoring for up to $30 \mathrm{~km}$ ). All the measurements are made at normal pressure and controlled room temperature conditions, at the same calibration parameters, with a spatial averaging of $1.02 \mathrm{~m}$. Continuous measurements are performed to monitor the influence of hydrogen outgassing on the Raman response of the saturated optical fibers. The duration of one acquisition is of $300 \mathrm{~s}$ and measurements are done every 30 min during the night whereas during the day an acquisition time of $30 \mathrm{~s}$ is used. All tests on irradiated fibers are done about one month after irradiation with 300s of acquisition time.

\subsection{Samples}

Five different types of commercial multimode fibers, showed in the table 2.1, are investigated: two types of Step Index Fibers (MMFSI), Radiation Resistant Fibers (RR-MMF) and Hydrogen Diffusion Resistant Fibers (HDR-MMF). We tested 4 different samples for each fiber type: a pristine sample (it means without any treatment, used as a reference), a hydrogenated sample and three not pre-treated $\gamma$-irradiated samples at the doses of $3 \mathrm{MGy}, 6 \mathrm{MGy}$ and $10 \mathrm{MGy}$ (10MGy is the accumulated dose in 100 years in the storage cells). Each sample of first four fibers consists about $100 \mathrm{~m}$ ( $83 \mathrm{~m}$ for Fiber IV) coiled with $8 \mathrm{~cm}$ of diameter. For the Fiber V we tested only $3 \gamma$-irradiated samples of 30m.

\subsection{Hydrogen loading and desorption condition}

Hydrogen is known to diffuse into silica. Loading durations can be calculated to achieve target hydrogen concentrations into the optical fiber core.

For the experiment, the samples were placed into hydrogen pressurized chambers at pressure of 202 bars and $80^{\circ} \mathrm{C}$ of temperature during 62 hours to achieve the saturation of the samples. Then samples were left at ambient condition and hydrogen desorbed naturally. All measurements on $\mathrm{H}_{2}$ loaded fibers were performed at room temperature to monitor the response of the instrument at constant ambient temperature as a function of the $\mathrm{H}_{2}$ concentration into the fiber core. For Cigéo project, when ventilation stops in storage cells, hydrogen content with slowly increases, to reach $100 \%$ in various months resulting in high $\mathrm{H}_{2}$ levels in the fibers used for sensing. 


\subsection{Irradiation conditions}

All fibers were gamma-irradiated with the Brigitte ${ }^{60} \mathrm{Co}$ facility of SCK $• \mathrm{CEN}$ to accumulate doses of $3 \mathrm{MGy}$, $6 \mathrm{MGy}$ and 10 MGy. Post mortem (one month after irradiation) temperature measurements were performed on pristine and on three irradiated $100 \mathrm{~m}$ samples of all fiber types. This fiber length is sufficient to obtain a clear evaluation of the radiation or hydrogen induced effects with our setup performances.

Table 2.1. Names and characteristics of the fiber samples. MMF-SI: Step Index multimode Fibers; RR-MMF: radiation Resistant Multimode Fibers; HDR-MMF: Hydrogen Diffusion Resistant Multimode Fibers.

\begin{tabular}{|c|c|c|c|c|c|}
\hline $\mathbf{N}^{\circ}$ Fiber & Type & Core & Cladding & Coating & Length \\
\hline Fiber I & MMF-SI & $50 \mu \mathrm{m}$ & $125 \mu \mathrm{m}$ & $\begin{array}{l}\text { Acrylate } \\
250 \mu \mathrm{m}\end{array}$ & $100 \mathrm{~m}$ \\
\hline Fiber II & MMF-SI & $\begin{array}{c}\text { Pure } \mathrm{SiO}_{2} \\
50 \mu \mathrm{m}\end{array}$ & $\begin{array}{c}\mathrm{SiO}_{2} \\
125 \mu \mathrm{m}\end{array}$ & $\begin{array}{c}\text { Polyimide } \\
155 \mu \mathrm{m}\end{array}$ & $100 \mathrm{~m}$ \\
\hline Fiber III & RR-MMF & $\begin{array}{c}\mathrm{SiO}_{2} \\
50 \mu \mathrm{m}\end{array}$ & $\begin{array}{l}\mathrm{F}-\mathrm{SiO}_{2} \\
125 \mu \mathrm{m}\end{array}$ & $\begin{array}{l}\text { UV cured resin } \\
245 \mu \mathrm{m}\end{array}$ & $100 \mathrm{~m}$ \\
\hline Fiber IV & HDR-MMF & $\begin{array}{c}\text { Doped } \mathrm{SiO}_{2}(\mathrm{P} \text { presence }) \\
50 \mu \mathrm{m}\end{array}$ & $125 \mu \mathrm{m}$ & $\begin{array}{l}\text { Carbon } \\
145 \mu \mathrm{m}\end{array}$ & $83 m$ \\
\hline Fiber V & RR-MMF & $\begin{array}{l}\mathrm{F}-\mathrm{SiO}_{2} \\
50 \mu \mathrm{m}\end{array}$ & $\begin{array}{l}\mathrm{F}-\mathrm{SiO}_{2} \\
125 \mu \mathrm{m}\end{array}$ & Acrylate & $30 \mathrm{~m}$ \\
\hline
\end{tabular}

\section{EXPERIMENTAL RESTULTS}

\subsection{Hydrogen loading and $\boldsymbol{\gamma}$-radiation effects on Raman sensor}

Figure 3.1a illustrates the strong negative impact of hydrogen loaded fiber I on the temperature estimation provided by the Raman instrument. Similar results were observed on fibers II, III, V. As shown in figure 3.1a, it is not possible to measure faithfully the temperature on sensors in fibers containing hydrogen, even low concentrations. For Fiber I, we observe an increase of temperature (up to the temperature limits of $800^{\circ} \mathrm{C}$ for this instrument) with the distance along the fiber placed at constant temperature. We observe that the temperature error increases with the distance and with the hydrogen content in the fiber. This is attributed to the differential attenuation $\left(\alpha_{a s}-\alpha_{s}\right)$ caused by the hydrogen related absorption bands ${ }^{5}$.

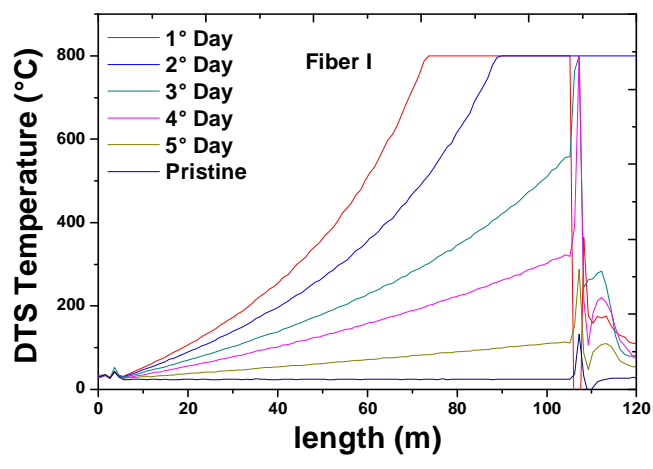

Figure 3.1a. Distributed Temperature measurements calculated by the instrument on pristine and $\mathrm{H}_{2}$-loading standard multimode fiber (Fiber I). The external temperature is around $25^{\circ} \mathrm{C}$.

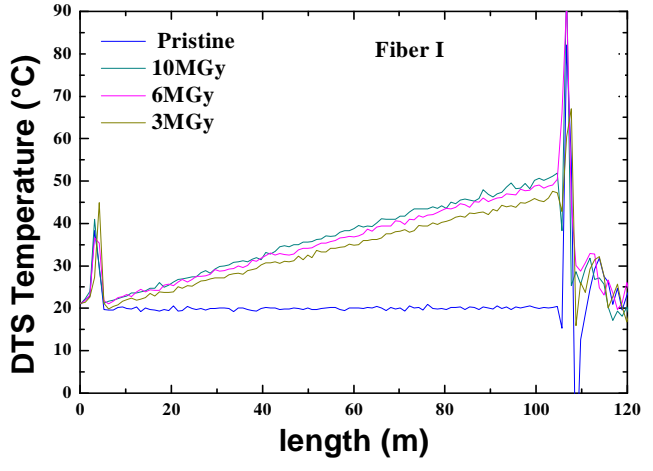

Figure 3.1b. Distributed Temperature measurements calculated by the instrument on Fiber I pristine and $\gamma$ - irradiated at different doses (3, 6 and $10 \mathrm{MGy})$. The external temperature is around $20^{\circ} \mathrm{C}$. 
Figure 3.1b illustrates the $\gamma$-rays impact on the Raman measurements based on fiber I (representative of other fibers). Gamma radiations also lead the device to calculate wrong temperature values, even if the observed errors are lowered compared to the case of hydrogen. Similarly, we observe that the temperature error increases with the distance and with the $\gamma$-irradiation dose, confirming that this negative influence is related to the spectral dependence of the radiation induced attenuation.

\section{DISCUSSION}

We observed that the Raman response is strongly influenced by $\gamma$ radiation dose and by hydrogen diffusion into the fiber core. The strong modifications in propagation losses limit the sensor performances under these harsh conditions $\left(>750^{\circ} \mathrm{C}\right.$ of temperature estimation errors). To overcome these problems, we selected carbon coating fibers to avoid the diffusion of hydrogen. Figure 3 illustrates the comparison between a Hydrogen diffusion resistant fiber with carbon coating (H-DR, Fiber IV) and a standard MMF (Fiber I). Without appropriate protection like carbon coating, Raman sensors cannot be directly used in $\mathrm{H}_{2}$ presence. At the opposite, the use of radiation resistant fibers to perform Raman distribute temperature measurements may be not sufficient. Figure 4 shows a radiation resistant fiber (RR-MMF, Fiber V) and a standard MMF (Fiber I). In the worst condition, 10MGy, on 30 meter we observe that the error on DTS temperature measure is reduced using a radiation resistant fiber than a standard MMF fiber. If the use of RRMMF permits to limit the RIA and then permits to work with great lengths of fiber, the selected fiber should also present a spectral dependence of the RIA allowing to impact the same way both AS and S wavelengths.

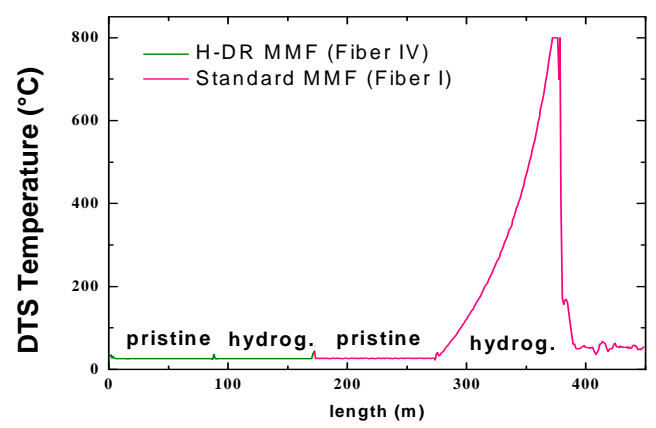

Figure 4.1a. Distributed Temperature measurements calculated by the instrument on $\mathrm{H}_{2}$-diffusion resistant fiber IV and on standard fiber I (pristine or loaded with $\mathrm{H}_{2}$ ).

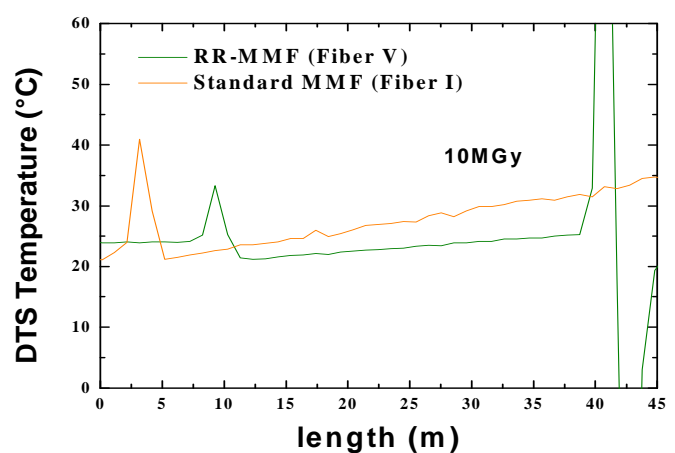

Figure 4.1b. Distributed Temperature measurements calculated by the instrument on radiation resistant and on standard MMF both irradiated at $10 \mathrm{MGy}$. The external temperature is around $22^{\circ} \mathrm{C}$ ).

\section{REFERENCES}

[1] X. Phéron, “ Durabilité des capteurs à fibres optiques sous environnement radiatif”, PhD Thesis, Université de SaintEtienne, 2013.

[2] Fernandez Fernandez, A., Rodeghiero, P., Brichard, B., Berghmans, F., Hargtog, A. H., Hughes, P., Williams, K. and Leach, A.P., "Radiation-Tolerant Raman Distributed Temperature Monitoring System for Large Nuclear Infrastructures", IEEE TRANSACTIONS ON NUCLEAR SCIENCE, 52(6), 2689-1694 (2005).

[3] Jensen, F. B. H., Takada, E., Nakazawa, M., Kakuta, T., Yamamoto, S., "Consequences of Radiation Effects on PureSilica-Core Optical Fibers Used for Raman-Scattering-Based Temperature Measurements", IEEE TRANSACTIONS ON NUCLEAR SCIENCE, 45(1), 50-58 (1998).

[4] http://www.sensornet.co.uk/

[5] Stone, J., "Interactions of hydrogen and deuterium with silica optical fibers: A Review", JOURNAL OF LIGHTWAVE TECHNOLOGY, LT5(5), 712-733 (1987). 